\title{
ALMACENAMIENTO DE ESPERMATOZOIDES EN LA VAGINA DE LA LAGARTIJA VIVÍPARA SCELOPORUS TORQUATUS (SAURIA: PRHYNOSOMATIDAE)
}

\author{
Martín MARTÍNEZ-TORRES \\ Laboratorio de Biología de la Reproducción-UMF, Facultad de Estudios Superiores Iztacala, Univer- \\ sidad Nacional Autónoma de México. Av. de los Barrios \# 1, Los Reyes Iztacala C. P. 54090, Tlalne- \\ pantla A. P. 314, Estado de México, MÉXICO. martor@servidor.unam.mx
}

Martínez-Torres, M. 2009. Almacenamiento de espermatozoides en la vagina de la lagartija vivípara Sceloporus torquatus (Sauria: Prhynosomatidae). Acta Zoológica Mexicana (n. s.), 25(3): 497-506. RESUMEN. Las hembras de muchas especies de lagartijas tienen la capacidad de almacenar espermatozoides en un sistema de túbulos especiales presentes en la vagina. Sin embargo, en otras especies los espermatozoides se almacenan en el infundíbulo. Se utilizó una técnica sencilla y rápida para determinar sí la vagina de Sceloporus torquatus tiene la capacidad de almacenar espermatozoides. En las hembras de esta especie, los espermatozoides son retenidos al menos 167 días en un sistema de túbulos especiales localizados en la vagina. La cantidad de espermatozoides presentes en los lavados vaginales disminuyó gradualmente a partir de diciembre y su desaparición ocurrió en la preñez tardía. Los espermatozoides ascienden al útero e infundíbulo antes de que ocurra la ovulación, sin embargo, en estos órganos no existen estructuras especiales para almacenar espermatozoides.

Palabras clave: Retención de espermatozoides, reptiles, aparato reproductor

Martínez-Torres, M. 2009. Sperm storage in the vagina of the viviparous lizard Sceloporus torquatus (Sauria:Prhynosomatidae). Acta Zoológica Mexicana (n. s.), 25(3): 497-506.

ABSTRACT. Females of many lizard species are able to store sperm in a special system of tubules present in the vagina. However, in other species sperm storage occurs in the infundibulum. A quick and simple technique to determine if the vagina of Sceloporus torquatus has the capacity to store sperm was used. In this species the spermatozoa are retained at least during 167 days after mating in a special system of tubules located in the vagina. The amount of spermatozoa observed through vaginal flushing was reduced gradually from December on and the spermatozoa disappeared in late pregnancy. The spermatozoa reached the uterus and infundibulum before ovulation started; however, in these organs there are no special structures for sperm storage.

Key words: Sperm retention, reptiles, reproductive tract

\section{INTRODUCCIÓN}

Las hembras de muchas especies de reptiles tienen la capacidad de almacenar espermatozoides en alguna región del aparato reproductor (Sever \& Hamlett 2002). Particularmente se ha observado que entre los pliegues de la vagina de diversos

Recibido: 26/06/2008; aceptado: 12/08/2009. 
saurios se presenta un sistema de túbulos especiales para almacenar espermatozoides (STEAE) (Saint-Girons 1962, Fox 1963, Cuellar 1966a). Sin embargo, en algunas especies de lagartijas (Phyllodactylus homolepidurus, Coleonyx variegatus, Cuellar 1966a) y serpientes (Thamnophis sirtalis, Hoffman \& Winsatt 1972, Agkistrodon piscivorus, Siegel \& Sever 2008) se ha observado que en el infundíbulo también existe este STEAE. En cambio, en las tortugas los espermatozoides se almacenan en túbulos ubicados en la región secretora del albumen y en el útero (Gopherus polyphemus, Palmer \& Guillette 1988, Sternotherus odoratus, Chrysemys picta, Trachemys scripta, Gist \& Congdon 1998) mientras que en el caimán (Alligator mississipiensis) la hembra almacena espermatozoides en la unión del útero y la vagina así como en el límite de la región del istmo (Gist et al. 2008). En los saurios, el tiempo de almacenamiento de espermatozoides puede variar desde cuatro semanas, como en Eumeces egregios (Shaefer \& Roeding 1973) hasta nueve meses, como en Chamaeleo hoehnelii (Jun-Yi 1982).

Los métodos utilizados por diversos investigadores para determinar la capacidad de las hembras de reptiles para retener espermatozoides son fundamentalmente dos y consisten en: (1) mantenerlas aisladas de machos y observar el número de puestas o camadas fértiles (Cuellar 1966b, Jun-Yi 1982, Villaverde \& Zucker 1998) y (2) sacrificarlas a diferentes tiempos durante la gestación y realizar cortes histológicos de la vagina y/o del infundíbulo y determinar la presencia de espermatozoides (Cuellar 1966a, Shaefer \& Roeding 1973, King 1977, Adams \& Cooper Jr. 1988).

En este estudio se utilizó una técnica rápida y sencilla para detectar la presencia de espermatozoides en la vagina de las hembras de Sceloporus torquatus. Además, se describe la estructura histológica del sistema de túbulos especiales para el almacenamiento de espermatozoides en la vagina de esta especie.

\section{MATERIAL Y MÉTODOS}

Trabajo de campo. Se capturaron 16 hembras adultas de $S$. torquatus (longitud hocico-cloaca $(\mathrm{mm}): 8.5 \pm 13$ D.E.; peso corporal (g): $25.17 \pm 3.6$ D.E.) en Cerro Gordo, San Juan Teotihuacán (19 45 3" N; 98 , 49 7' W) durante la tercera semana de septiembre $(n=4)$, octubre $(n=5)$ noviembre $(n=4)$ y diciembre $(n=3)$ de 2003.

Una vez colectadas, las hembras fueron marcadas por el método de ectomización de falanges e inmediatamente después se procedió a lavar la vagina de los organismos (Fig. 1) usando una pipeta Pasteur con aproximadamente 2001 de solución salina fisiológica para reptil (cloruro de sodio al $0.7 \%$, Guillette 1982). La punta de la pipeta Pasteur se introdujo por la cloaca y se dirigió hacia la vagina; una vez ubicada en este lugar se bombeó 3 a 4 veces la solución e inmediatamente después se recuperó una alícuota y se depositó en un portaobjetos. Se agregó una gota de yodo-lugol a la muestra y se procedió a observarla a 10X y 40X para determinar la presencia de espermatozoides. En aquellas hembras en las cuales se observaron 


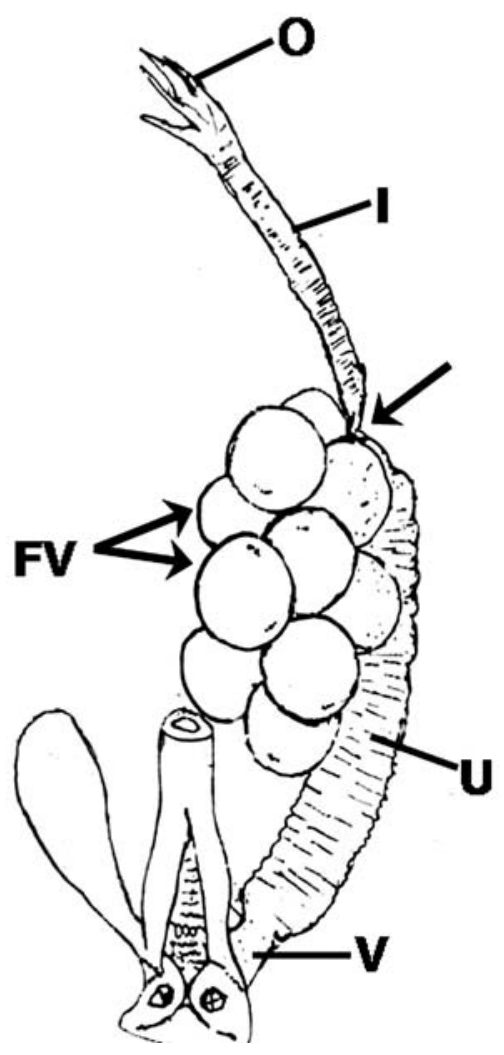

Figura 1. Esquema del aparato reproductor de una hembra adulta de Sceloporus torquatus en la etapa de vitelogénesis avanzada: Vagina (V), útero (U), límite útero-infundíbulo, ('); infundíbulo (I), ostium (O) y folículos vitelogénicos (FV).

espermatozoides se revisó una segunda alícuota sin yodo-lugol para determinar si presentaban movilidad. Solamente una lagartija por mes, de las que presentaron espermatozoides, fue llevada al laboratorio para continuar con la revisión de manera periódica. El resto de los organismos fueron liberados una vez efectuados los lavados.

Trabajo de laboratorio. Un total de cuatro hembras fueron mantenidas en cautiverio en terrarios de $136 \times 89 \times 54 \mathrm{~cm}$, con temperatura y fotoperiodo natural y con acceso libre a alimento (larvas de Tenebrio, palomillas, grillos domésticos y chapulines) y agua. Estas lagartijas se sometieron a lavados vaginales cada dos semanas para determinar el tiempo de retención de espermatozoides y una de estas 
hembras se sacrificó (25 de noviembre) para determinar la presencia de espermatozoides en el útero y en el infundíbulo mediante el lavado de estas estructuras, así como para establecer las características histológicas del sitio de almacenamiento de espermatozoides.

La hembra se sacrificó con una sobredosis de pentobarbital sódico vía intracardiaca y, previo a la fijación de la vagina, el útero y el infundíbulo derecho en el límite útero-vagina y útero-infundíbulo se ligaron con hilo nylon de 5 ceros (Fig. 1) e inmediatamente después fueron disecados y colocados en solución fisiológica fría $\left(4^{\circ} \mathrm{C}\right)$ para reptil. Se tomó el segmento uterino y se introdujo (por el lado de la ligadura) en la cavidad de este órgano la punta de la aguja de una jeringa insulínica que contenía 5001 de solución fisiológica para reptil. En seguida se perfundió el líquido dentro de la cavidad y se recuperó el fluido por el lado opuesto de donde se insertó la aguja. El mismo procedimiento se aplicó al infundíbulo. Los lavados (del útero e infundíbulo) fueron colocadas en un portaobjeto y se agregó una gota de yodo-lugol e inmediatamente después fueron observadas al microscopio para determinar la presencia de espermatozoides.

Histología. Inmediatamente después que fueron removidos el útero y el infundíbulo derecho, se procedió a disecar la vagina (de ambos lados), así como un segmento de la parte media del útero y otro del infundíbulo izquierdo (de alrededor de $5 \mathrm{~mm}$ de longitud cada uno) y se fijaron en formol al $10 \%$ amortiguado durante 24 horas. Después de la fijación los tejidos se deshidrataron con alcoholes graduales hasta etanol absoluto, se aclararon en xilol y se incluyeron en Paraplast. Se obtuvieron cortes de $7 \mathrm{~m}$ de grosor, se desparafinaron y se tiñeron con hematoxilina y eosina (Luna 1968). Finalmente se montaron con resina sintética, se observaron al microscopio para determinar la presencia del STEAE en la vagina, útero o infundíbulo y/o de espermatozoides.

\section{RESULTADOS}

Espermatozoides en los lavados vaginales. En las hembras colectadas en septiembre no se observaron espermatozoides en los lavados vaginales, en cambio sí se detectaron en todas las hembras capturadas a partir del 20 de octubre. Los espermatozoides contenidos en los lavados vaginales de octubre y noviembre mostraron movilidad y se presentaron dispersos en la solución fisiológica (Fig. 2). A partir de diciembre se observaron espermatozoides inmóviles en los lavados vaginales. También se detectó una disminución gradual de estas células en los lavados a partir de diciembre hasta abril 4. A partir de esta fecha ya no se observaron espermatozoides en ninguno de los lavados vaginales, por lo que el tiempo de almacenamiento de espermatozoides en esta especie es de al menos 167 días.

Espermatozoides en lo lavados de útero e infundíbulo. En los lavados de útero e infundíbulo también se detectaron espermatozoides, sin embargo, no se contaron. 


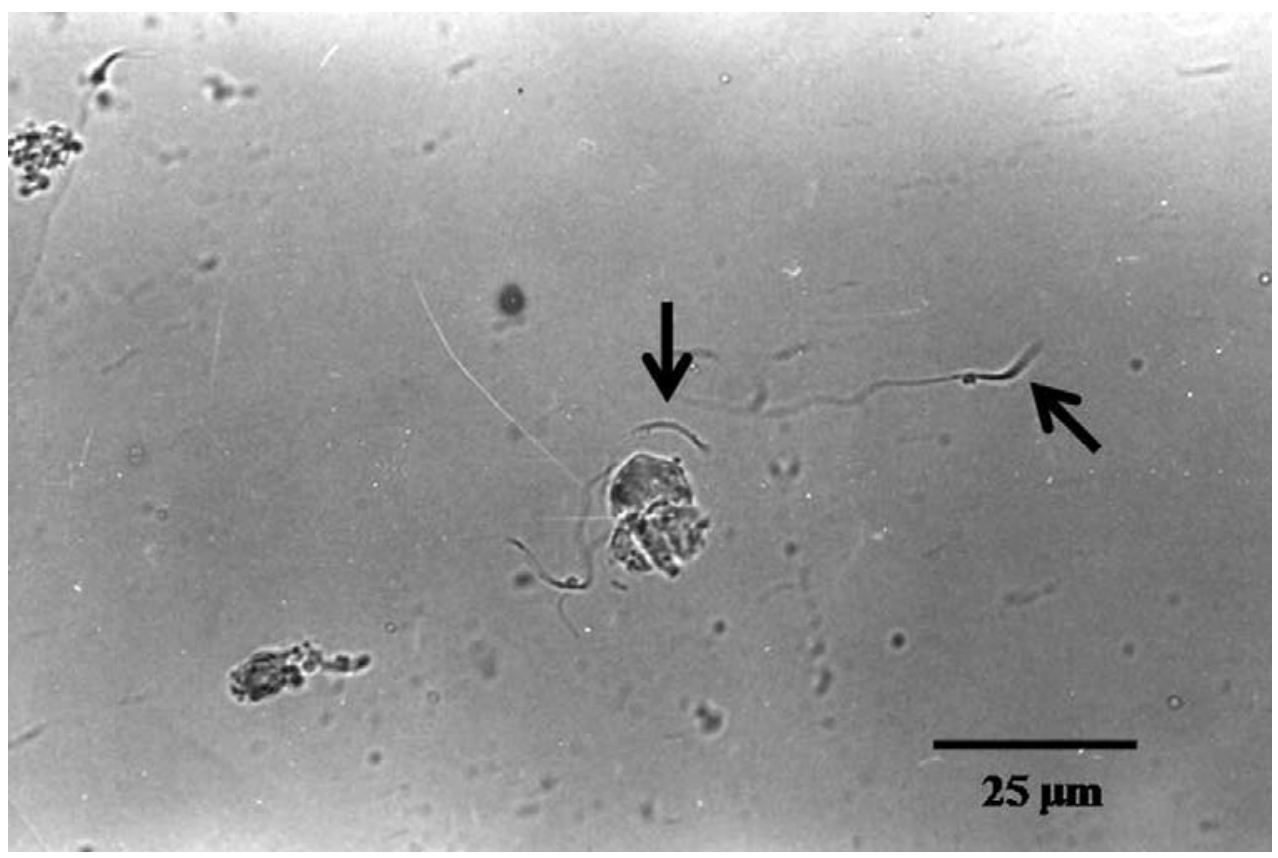

Figura 2. Espermatozoides $(\rightarrow$ ) de Sceloporus torquatus obtenidos por lavado de la vagina en la última semana de noviembre.

Los espermatozoides se observaron íntegros, pero no se pudo determinar su movilidad ya que se agregó yodo-lugol para observarlos y esta solución los mata.

Histología. En los cortes histológicos de la vagina se observó la presencia de receptáculos seminales conteniendo espermatozoides. Estas estructuras se caracterizan por tener una mucosa con pliegues ramificados y limitados por epitelio cilíndrico simple ciliado. Los espermatozoides fueron observados tanto en la luz de la vagina como en los pliegues (Fig. 3A). Además, en algunos lugares de estos receptáculos, se observó que las cabezas de los espermatozoides estaban en contacto con las células epiteliales (Fig. 3B).

No se observaron receptáculos seminales en los cortes histológicos del útero (Fig. 3C) ni del infundíbulo (Fig. 3D). Sin embargo, se detectaron abundantes espermatozoides libres en la luz del útero, cerca del epitelio. Un conteo al microscopio demostró más de cien espermatozoides en la cavidad uterina, muy cerca uno de otro (Fig. 3C). En cambio en el infundíbulo fueron escasos, ya que se observaron sólo cuatro cabezas de espermatozoides en los cortes histológicos de este órgano (Fig. 3D). 


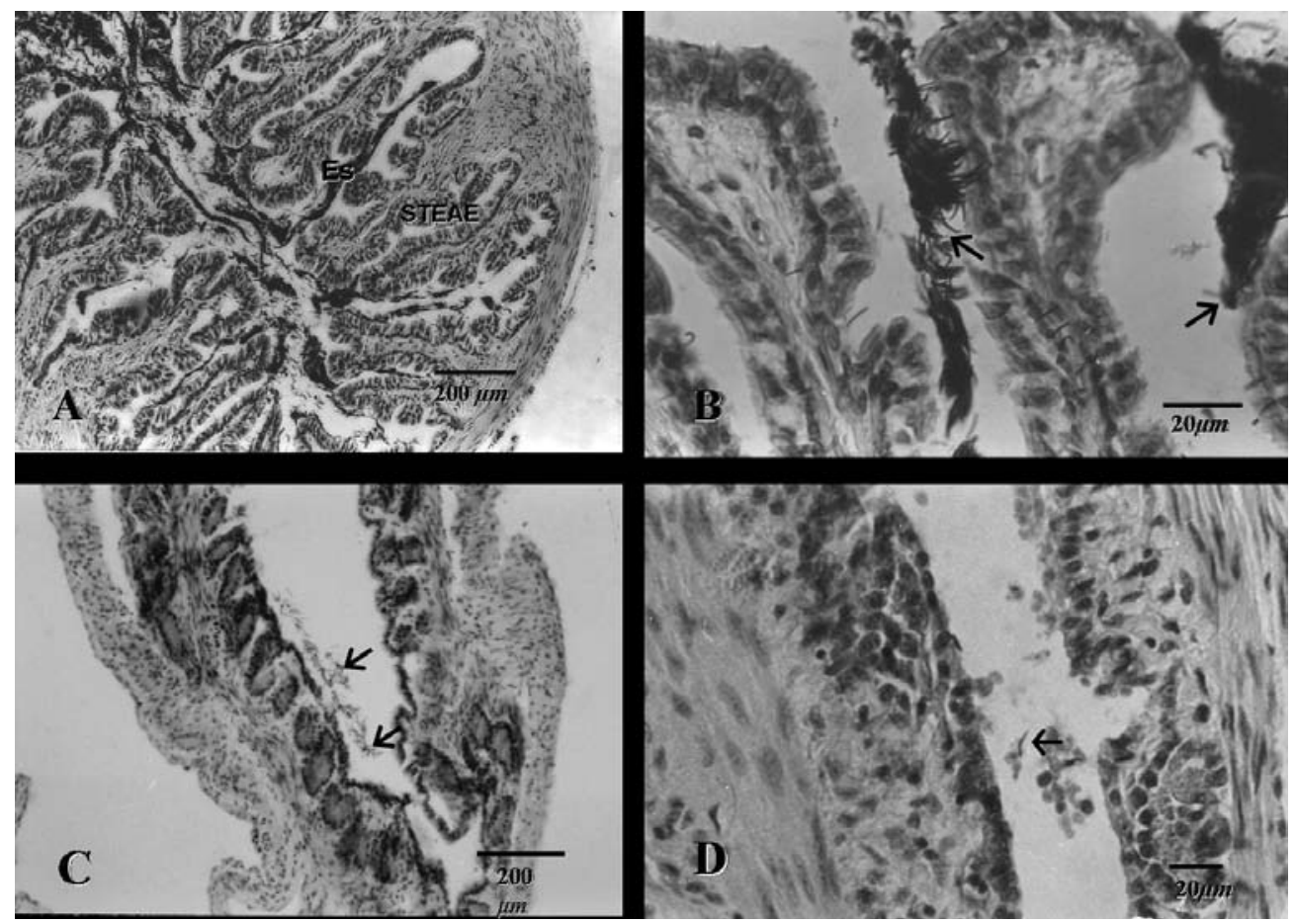

Figura 3. Cortes histológicos del aparato reproductor de una hembra de Sceloporus torquatus. (A) Corte histológico de la vagina. Se observan espermatozoides (Es) en la luz de la cavidad así como en el sistema de túbulos especiales para el almacén de espermatozoides (STEAE). (B) Cabezas de espermatozoides $(\rightarrow)$ en estrecho contacto con las células epiteliales del STEAE. (C) Corte histológico del útero. Las flechas señalan la presencia de acúmulos de espermatozoides libres en la cavidad $(\rightarrow)$. La mucosa uterina carece de un STEAE. (D) Corte histológico del infundíbulo. Las flechas señalan algunas cabezas de espermatozoides libres en la cavidad.

\section{DISCUSIÓN}

En este estudio se encontró que las hembras de $S$. torquatus retienen espermatozoides en receptáculos seminales presentes en la vagina desde el momento del apareamiento hasta el último tercio de la gestación. Los receptáculos donde estas lagartijas almacenan a los espermatozoides presentan características histológicas similares al STEAE de Anolis carolinensis descrito por Fox (1963). De esta manera, los receptáculos seminales donde las hembras de $S$. torquatus almacenan espermatozoides constituyen un verdadero STEAE.

El almacenaje de espermatozoides es una estrategia reproductora que permite el encuentro de los gametos (espermatozoides y ovocitos) en aquellas especies que: a) 
presentan ciclo reproductor asincrónico entre los sexos (Cuellar 1966b, Smyth \& Smith 1968, Birkhead \& Moller 1993); b) los machos presentan baja movilidad o hábitos solitarios (Bustard 1965); c) las hembras presentan una estación reproductora prolongada o múltiples puestas (Conner \& Crews, 1980) y d) presentan desigualdad en la proporción de sexos (menos machos que hembras) (Jun-Yi, 1982). S. torquatus es una lagartija vivípara que presenta un ciclo reproductor estacional anual asincrónico entre hembras y machos, por lo que en esta especie el almacenaje de espermatozoides es una estrategia obligada para que pueda ocurrir la fecundación. Feria-Ortiz et al. (2001) observaron un intento de apareamiento en octubre en una población del Pedregal de San Ángel de la Ciudad de México de S. torquatus y en este estudio se detectaron espermatozoides a partir de la última semana de ese mes. Estas observaciones confirman que en esta lagartija el apareamiento ocurre en octubre y que las hembras almacenan espermatozoides más allá del momento de la fecundación, ya que estos gametos fueron observados en lavados vaginales en la preñez tardía (4 de abril). En esta especie los nacimientos ocurren a finales de abril (Guillette \& Méndez-De la Cruz 1993).

Después de la cópula los espermatozoides entran en un ambiente donde las condiciones están determinadas por las secreciones del tracto genital de la hembra. En diversas especies de lagartijas (Acanthodactylus scutellatus, Bou-Resli et al. 1981; Anolis sagrei, Sever \& Hamlett 2002), serpientes (Thamnophis sirtalis, Hoffman \& Wimsatt 1972, Seminatrix pygaea Sever \& Ryan 1999) tortugas (Sarkar et al. 2003) y cocodrilos (Gist et al. 2008) existen células secretoras en los STEAE. Saint-Girons (1975) sugirió que en los reptiles estas secreciones son utilizadas como nutrientes por los espermatozoides y, además, que su retención en la vagina es necesaria para que los espermatozoides maduren. En el STEAE de $S$. torquatus no se observaron células secretoras, únicamente se observaron células ciliadas, sin embargo, se desconoce si estas células liberan azúcares o alguna otra sustancia que permita la sobrevivencia de los espermatozoides por más de 5 meses. Por otro lado, en los cortes histológicos de la vagina de $S$. torquatus se observó que los espermatozoides están en estrecho contacto con las células del epitelio vaginal. Esta condición sugiere que puede existir una interacción (por ejemplo, captación de nutrientes del glucocalix de las células epiteliales) que permita que la viabilidad de los espermatozoides se mantenga por tiempos largos. En el hámster (Smith \& Yanagimachi 1990, 1991) se ha observado que los espermatozoides que permanecen unidos a las células epiteliales tienen mayores tasas de sobrevivencia que aquellos que están libres en la cavidad. Aunque una situación similar a la que aquí se describe en $S$. torquatus ha sido observada en otras lagartijas (Eumeces egregius, Shaefer \& Roeding 1973; Acanthodactylus scutellatus, Bou-Resli et al. 1981; Holbroquia propinqua, Adams \& Cooper 1988) se desconoce sí en los reptiles existe una situación similar a la que ocurre en los mamíferos. 
Los mecanismos que regulan la liberación de espermatozoides del STEAE y la migración a las regiones superiores del oviducto han sido poco estudiadas en los reptiles. Halpert et al. (1982) sugirieron que en Tamnophis sirtalis las secreciones del epitelio vaginal facilitan el transporte de los espermatozoides al infundíbulo y en $S$. torquatus podría ocurrir una situación similar. En los mamíferos (ratón, rata, toro, conejo) es ampliamente conocido que las secreciones del útero y del oviducto estimulan el metabolismo del espermatozoide (hiperactivación, capacitación etc.) (Chang 1951, Hogart 1978, Suarez 2008). Estos aspectos tampoco han sido estudiados en los reptiles y además se desconoce la cantidad de espermatozoides que transitan por la cavidad uterina, si viajan de manera individual o en acúmulos. También se desconoce el número de espermatozoides que llegan al infundíbulo. Los resultados del análisis histológico de este estudio con $S$. torquatus sugieren que algunos cientos de espermatozoides alcanzan el útero. Es muy posible que transiten a través del útero formando acúmulos, pues en los cortes histológicos se observó que los espermatozoides estaban muy cerca unos de otros. En cambio, en los cortes histológicos del infundíbulo la presencia de espermatozoides fue mínima, sugiriendo que solamente decenas alcanzan esta región del oviducto.

AGRADECIMIENTOS. A Gloria Sánchez por su apoyo en la colecta de los organismos y mi mayor agradecimiento a los revisores anónimos por el gran esfuerzo y entusiasmo al revisar este manuscrito.

\section{LITERATURA CITADA}

Adams, C. S. \& W. E. Cooper Jr. 1988. Oviductal morphology and sperm storage in the keelep earless lizard, Holbrookia propinqua. Herpetologica, 44: 190-197.

Birkhead, T. R. \& A. P. Moller. 1993. Sexual selection and the temporal separation of reproductive events: sperm storage data from reptiles, birds and mammals. Biological Journal of the Linnean Society, 50: 295-311

Bou-Resli, M. N., N. L. F. Bishay \& N. S. Al-Zaid. 1981. Observations on the fine structure of the sperm storage crypts in the lizard Acnthodactylus scutellatus hardyi. Archives du Biologie (Bruxelles), 92: 287-298.

Bustard, H. R. 1965. Observations of the life history and behavior of Chamaleo hohnelli (Steindachner). Copeia, 1965: 401-410.

Chang, M. C. 1951. Fertilizing capacity of spermatozoa deposited into the fallopian tubes. Nature, 168 : 697-698.

Conner, J. \& D. Crews. 1980 Sperm transfer and storage in the lizard, Anolis carolinensis. Journal of Morphology, 163: 331-348.

Cuellar, O. 1966a. Oviductal anatomy and sperm storage structures in lizards. Journal of Morphology, 119:7-20.

Cuellar, O. 1966b. Delayed fertilization in the lizard Uta stansburiana. Copeia 1966:549-552.

Feria-Ortiz, M., A. Nieto-Montes de Oca \& I. H. Salgado-Ugarte. 2001. Diet and reproductive biology of the viviparous lizard Sceloporus torquatus torquatus (Squamata: Phrynosomatidae). Journal of Herpetology, 31: 104-112.

Fox, W. 1963. Special tubules for sperm storage in female lizards. Nature, 196: 500-501. 
Gist, D. H. \& J. D. Congdon. 1998. Oviductal sperm storage as a reproductive tactic of the turtles. Journal of Experimental Zoology, 282: 526-534.

Gist, D. H., A. Bagwill, V. Lance, D. M. Sever \& R. M. Elsey. 2008. Sperm storage in the oviduct of the American alligator. Journal of Experimental Zoology Part A, Ecology Genetics and Physiology, 309: 581-587.

Guillette, L. J. Jr. 1982. A physiological (Ringer's) solution for anoline lizard. Herpetological Review, 13: 37-38.

Guillette, L. J. Jr. \& F. R. Méndez-De la Cruz. 1993. The reproductive cycle of the viviparous Mexican lizard Sceloporus torquatus. Journal of Herpetology, 27: 168-174.

Halpert, A. P., W. R. Garstka \& D. Crews. 1982. Sperm transport and storage and its relation to the annual sexual cycle of the female red sided garter snake, Thamnophis sirtalis parietalis. Journal of Morphology, 174: 149-159.

Hoffman, L. H. \& W. A. Wimsatt. 1972. Histochemical and electron microscopy observations on the sperm receptacles in the garter snake oviduct. American Journal of Anatomy, 134: 71-96.

Hogarth, P. J. 1978. Sperm in the female tract. Pp. 36-48. In: Robert Mac Lehose (Ed). Biology of Reproduction. Halsted Press, John Wiley and Sons. New York. Robert MacLehose.

Jun-Yi, L. 1982. Sperm retention in the lizard Chamaeleo hoehnellii. Copeia 1982: 488-489.

King, M. 1977. Reproduction in the australian gekko Phyllodactylus marmoratus (Gray). Herpetologica, 33: 7-13.

Luna, G. L. 1968. Manual of Histological Staining Methods of the Armed Forces Institute of Pathology. Mc Graw-Hill Book Co., New York.

Palmer, B. D. \& J. L. Guillette Jr. 1988. Histology and functional morphology of the female reproductive tract of the tortoise Gopherus polyphemus. American Journal of Anatomy, 183: 200-211.

Saint-Girons, H. 1962. Presence of receptacles seminaux chez les camaeons. Beaufortia, 9: 165-172.

Saint-Girons, H. 1975. Sperm survival and transport in the female genital tract of reptiles. Pp. 105-113. In: E. S. E. Hafez and C. G. Thibault (Eds.). Biology of spermatozoa. S. Karger, A. G. Basel, Switzerland.

Sarkar, S., N. K. Sarkar \& B. R. Maiti. 2003. Oviductal sperm storage structure and their changes during the seasonal (dissociated) reproductive cycle in the soft-shelled turtle Lissemys punctata. Journal of Experimental Zoology A, Comparative Experimental Biology, 295: 83-91.

Sever, D. M. \& T. J. Ryan. 1999. Ultrastructure of the reproductive system of the black swam snake (Seminatrix pygaea). Part I. Evidence for oviductal sperm storage. Journal of Morphology, 241: 118.

Sever, D. M. \& W. C. Hamlett. 2002. Female sperm storage in reptiles. Journal Experimental Zoology Part A: Comparative Experimental Biology, 292: 187-199.

Shaefer, G.C. \& C.E. Roeding. 1973. Evidence for vaginal storage in the mole skink, Eumeces egregius. Copeia, 1973: 346-347.

Siegel, D. S. \& Sever D. M. 2008. Seasonal variation in the oviduct female Agkistrodon piscivorus (Reptilia: Squamata): an ultrastructural investigation. Journal of Morphology, 269: 980-997.

Smyth, M. \& M. J. Smith 1968. Obligatory sperm storage in the skink Hemigergis peronii. Science, 161: $575-575$

Smith, T. T. \& R. Yanagimachi. 1990. The viability of hamster spermatozoa stored in the isthmus oviduct: the importance of sperm-epithelial contact for sperm survival. Biology of Reproduction, 42:450-457.

Smith, T. T. \& R. Yanagimachi. 1991. Attachment and release of spermatozoa from the caudal itsmo of hamster oviduct. Journal Reproduction and Fertility, 91:567-573. 
Martínez-Torres: Almacenamiento de espermatozoides en lagartijas

Suarez, S. S. 2008. Regulation of sperm storage and movement in the mammalian oviduct. International Journal of Developmental Biology, 52: 455-462.

Villaverde, G. A. \& N. Zucker. 1998. Sperm storage resulting in viable offspring in the tree lizard Urosaurus ornatus (Sauria: Phrynosomatidae). Southwestern Naturalist, 43: 92-95. 